\title{
Beating Your Fractional Beatty Game Opponent and: What's the Question to Your Answer?
}

\author{
Aviezri S. Fraenkel* \\ Department of Computer Science and Applied Mathematics \\ Weizmann Institute of Science \\ Rehovot 76100, Israel
}

November 30, 2011

To Herb Wilf on his 80th birthday: He shall be as a CW tree planted by the waters that spreads out its roots by the river, shall not see when heat comes, its leaf shall remain green, shall not be anxious in the year of drought, nor shall it cease from bearing fruit (adapted from Jeremiah 17, 8).

\begin{abstract}
Given a subtraction game on two piles of tokens, the usual question is to characterize its $P$-positions. These normally split the positive integers into two nonintersecting sequences. Here we invert the problem: We are given two sequences, and the challenge is to find appropriate succinct game rules for a game having the given $P$-positions. The main additional challenge in this work is that the given sequences do not split the positive integers. We present two solutions for a seemingly first such problem, the second in terms of two exotic numeration systems. Both characterizations lead to polynomial-time winning strategies for the game induced by the two sequences.
\end{abstract}

\section{Prologue}

Preliminary Thoughts. Subtraction games, also called take-away games, are games on $m$ piles of tokens, where each of two players playing alternately, selects one or more piles and removes from them a number of tokens according to the specified game rules. ${ }^{1}$ In this paper we consider impartial subtraction games. For $m \geq 2$, the $P$-positions of such games typically split the positive integers into $m$ disjoint sets $A^{1}, \ldots, A^{m}: \cup_{i=1}^{m} A^{i}=\mathbb{Z}_{\geq 1}, A^{i} \cap A^{j}=\emptyset$ for all $i \neq j$. Two of many examples: [3], [6]. There are only a few studies where this splitting does

\footnotetext{
*fraenkel@wisdom.weizmann.ac.il http://www.wisdom.weizmann.ac.il/ fraenkel

${ }^{1}$ They can equivalently be modeled as games played on a collection of nonnegative integers, which are reduced by the players to 0 according to the game rules.
} 
not hold. In [2] and [8] the Nim move is restricted to taking any positive multiple of $b$ tokens from a single pile, where $b$ is an a priori given positive integer parameter (and there is a restricted Wythoff move in [8]). The $P$-positions there constitute $b$ pairs of integers and there are omissions and repetitions of integers in some of the pairs. Sequences that jointly cover every positive integer precisely $m$ times for any given $m \geq 1$ were given by O'Bryant [16] using a generating function approach; and Graham and O'Bryant [10] used them for generalizing a conjecture about splitting sets. They were constructed by elementary means by Larsson and applied there to combinatorial game theory [14]. More recently, Gurvich [11] considered a generalization of Wythoff's game where, for $m=2$, $A^{1} \cap A^{2}=\emptyset$, but $\left|\mathbb{Z}_{\geq 1} \backslash\left(A^{1} \cup A^{2}\right)\right|=\infty$. In [17] games are analyzed for which both $A^{1} \cap A^{2} \neq \emptyset$ and $\left|\mathbb{Z}_{\geq 1} \backslash\left(A^{1} \cup A^{2}\right)\right|=\infty$. But exceptions they are.

In the present paper we consider a case, also for $m=2$, apparently a first of its kind, where the $P$-positions constitute a single pair $\left(A^{1}, A^{2}\right)$ of integers, $\left|A^{1} \cap A^{2}\right|=\infty$, but $A^{1} \cup A^{2}=\mathbb{Z}_{\geq 1}$. The easy part is to construct $A^{1}, A^{2}$ with such properties; the hard part is to formulate appropriate succinct game rules for a game whose $P$-positions are such non-complementary sequences. We seek a question for a given answer!

\section{The Game, Main Theorem and Examples}

Denote by $\varphi=(1+\sqrt{5}) / 2$ the golden section. Then $\varphi^{2}=(3+\sqrt{5}) / 2$, and $\varphi^{-1}+\varphi^{-2}=1$. Multiplying by $3 / 2$, we get

$$
\alpha^{-1}+\beta^{-1}=3 / 2,
$$

where

$$
\alpha=\frac{2 \varphi}{3}=\frac{1+\sqrt{5}}{3}=1.0786893 \ldots, \quad \beta=\frac{2 \varphi^{2}}{3}=\frac{3+\sqrt{5}}{3}=1.745356 \ldots,
$$

and $\beta-\alpha=2 / 3$. For $n \geq 0$, let $a_{n}=\lfloor n \alpha\rfloor, b_{n}=\lfloor n \beta\rfloor$. Let

$$
A:=\cup_{n \geq 0} a_{n}, \quad B:=\cup_{n \geq 0} b_{n}, \quad \mathcal{T}:=\cup_{n \geq 0}\left(a_{n}, b_{n}\right), a_{n} \in A, b_{n} \in B .
$$

We denote by $\overline{\mathcal{T}}=\mathbb{Z}_{\geq 0} \backslash \mathcal{T}$ the complement of $\mathcal{T}$, that is, all pairs $(x, y) \in$ $\mathbb{Z}_{\geq 0} \times \mathbb{Z}_{\geq 0}$ not in $\mathcal{T}$. Some of the first few terms of $A$ and $B$ are displayed in Table 1 .

Table 1. Excerpts of the first few terms of the sequences $A$ and $B$.

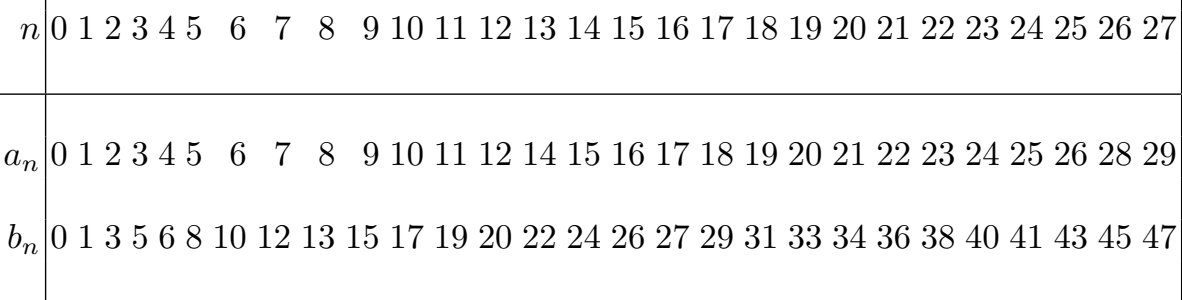




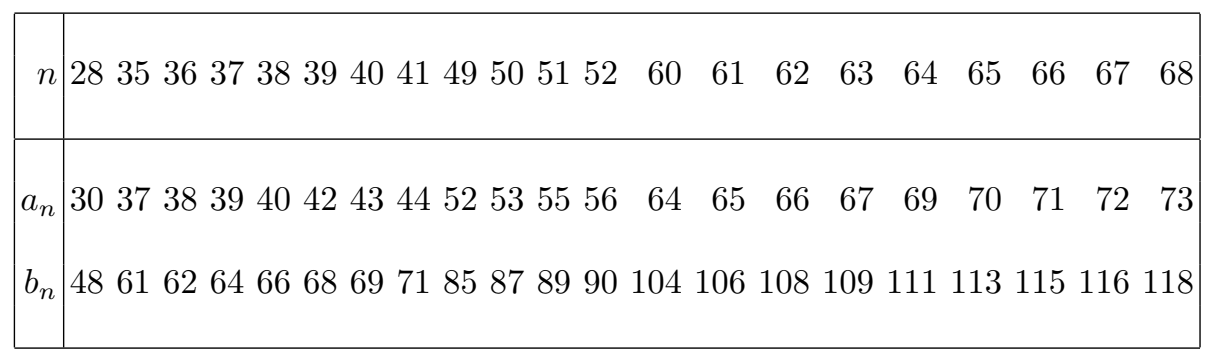

In the game FrEAK there are two piles of finitely many tokens. We denote the piles by the number of tokens they contain, i.e.,

$$
(x, y) \text {, with } 0 \leq x \leq y .
$$

Two players alternate in reducing the piles. Play ends when the piles are empty. The player first unable to move loses and the opponent wins (normal play).

Remark 1. In a move from a position $(x, y)$ subject to (2) where $x$ is unchanged, but $y \rightarrow y-t$ with $t>0$, we may have $x \leq y-t$ or $y-t<x$. To be consistent with (2) we write $(x, y) \rightarrow(x, y-t)$ in the former case, and $(x, y) \rightarrow(y-t, x)$ in the latter case.

The $P$-positions of FreAK are given, namely $\mathcal{P}=\mathcal{T}$. What are succinct game rules of FreAK such that it has precisely these $P$-positions?

We claim that at each stage a FrEAK player has the choice of making one of the following two types of moves:

(I) (Restricted Wythoff move.) $(x, y) \rightarrow(x-t, y-t)$ for every $t \in\{1, \ldots, x\}$, except that this move is blocked if $t \in\{1,2,3\}$ and $x \in A$ and $y \in B$.

(II) (Restricted Nim move.) (a) $(x, y) \rightarrow(x-t, y)$ for any $0<t \leq x$; or

(b) $(x, y) \rightarrow(x, y-t)$ for any $0<t \leq y$; or

(c) $(x, y) \rightarrow(y-t, x)$ for any $0<t \leq y$, except that this move is blocked if $x \in A \cap B$ and $y \in B$.

Theorem 1. For the game FrEAK, $\mathcal{P}=\mathcal{T}$.

Example 1. We refer the reader to Table 1.

- The moves from $\mathcal{T}$ to $\mathcal{T}(4,6) \rightarrow(3,5),(12,20) \rightarrow(11,19)$ are blocked because $4,12 \in A$ and $6,20 \in B((\mathrm{I}), t=1)$.

- Similarly, the moves $(14,22) \rightarrow(12,20),(28,45) \rightarrow(26,43)$ are blocked $((\mathrm{I}), t=2)$.

- Also $(14,22) \rightarrow(11,19),(43,69) \rightarrow(40,66)$ are blocked $((\mathrm{I}), t=3)$.

- $(12,20) \rightarrow(7,12)$ and $(19,31) \rightarrow(11,19)$ are blocked by (II)(c), since $12 \in A \cap B, 19 \in A \cap B$; and $20,31 \in B$. 
- For every $s>13,(13, s) \rightarrow(8,13)$ is not blocked by (II)(c), since $13 \notin A$.

- Notice that moves from the complement $\overline{\mathcal{T}}$ to $\mathcal{T}$ such as $(15,34) \rightarrow(15,24)$, $(15,22) \rightarrow(14,22)$ or $(10,17),(11,16) \rightarrow(8,13)$ are not blocked.

It should be clear that a winning strategy for FREAK can be effected by means of the $P$-positions. Given any game position $(x, y)$ subject to $(2)$, we have only to find out to which sequence, $A$ or $B, x$ and $y$ belong. The complexity of the implied computation will be discussed later on.

\section{Preliminaries}

For proving Theorem 1, we begin by collecting a few facts about the sequences $A$ and $B$.

For any number $r \in \mathbb{R}_{>0}$ and $n \in \mathbb{Z}_{\geq 0}$, let $\Delta\lfloor n r\rfloor=\lfloor(n+1) r\rfloor-\lfloor n r\rfloor$.

Lemma 1. (i) Each of the sequences $A$ and $B$ is strictly increasing.

(ii) For every $n \geq 0, \Delta\lfloor n \alpha\rfloor=2 \Longrightarrow \Delta\lfloor n \beta\rfloor=2$.

Proof. Note that $1<\alpha<\beta<2$. These inequalities imply:

$$
\Delta\lfloor n \alpha\rfloor \in\{1,2\}, \quad \Delta\lfloor n \beta\rfloor \in\{1,2\} \quad \text { for all } n \in \mathbb{Z}_{\geq 1} .
$$

Also note that $\Delta\lfloor n \alpha\rfloor=2$ if and only if $(n+1) \alpha=i+1+\delta_{1}, n \alpha=i-\delta_{2}$ for some integer $i=i(n)$, and $0<\delta_{1}, \delta_{2}<\alpha-1<0.08$. For such $n$ we have, $(n+1) \beta=(n+1)(\alpha+2 / 3)=i+1+\delta_{1}+2(n+1) / 3 ; n \beta=n(\alpha+2 / 3)=i-\delta_{2}+2 n / 3$. Put $n=3 k+i, i \in\{0,1,2\}$. Then $(n+1) \beta=i+1+\delta_{1}+2 k+2(i+1) / 3$, $n \beta=i-\delta_{2}+2 k+2 i / 3$. We consider three cases:

1. $i=0$. Then $\Delta\lfloor n \beta\rfloor=(i+2 k+1)-(i-1+2 k)=2$.

2. $i=1$. Then $\Delta\lfloor n \beta\rfloor=(i+2 k+2)-(i+2 k)=2$.

3. $i=2$. Then $\Delta\lfloor n \beta\rfloor=(i+2 k+3)-(i+2 k+1)=2$.

Thus $\Delta\lfloor n \alpha\rfloor=2 \Longrightarrow \Delta\lfloor n \beta\rfloor=2$. This implies,

$$
\lfloor n \beta\rfloor-\lfloor n \alpha\rfloor \text { is a nondecreasing function of } n \text {. }
$$

The properties (3) immediately imply (i). Let $\lfloor n \alpha\rfloor=K,\lfloor n \beta\rfloor=L$. If $\Delta\lfloor n \alpha\rfloor=$ 2 , then $\lfloor(n+1) \alpha\rfloor=K+2,\lfloor(n+1) \beta\rfloor=L+\delta$, where $\delta \in\{1,2\}$ by (3). Now $\lfloor n \beta\rfloor-\lfloor n \alpha\rfloor=L-K,\lfloor(n+1) \beta\rfloor-\lfloor(n+1) \alpha\rfloor=L-K+\delta-2$. By (4), $L-K+\delta-2 \geq L-K$, so $\delta \geq 2$. By (3), $\delta=2$, establishing (ii).

Corollary 1. For every $n \geq 0, \Delta\lfloor n \beta\rfloor=1 \Longrightarrow \Delta\lfloor n \alpha\rfloor=1$.

Proof. In view of (3), this is the contrapositive statement of Lemma 1(ii). 
Lemma 2. We have,

(i) $A \cup B=\mathbb{Z}_{\geq 0}$ (every nonnegative integer appears in $A \cup B$ ).

(ii) Every nonnegative integer $N$ is assumed at most twice in $A \cup B$. If $N$ appears twice, it appears once in $A$ and once in $B$.

(iii) $b_{m}=a_{n} \Longrightarrow m \leq n$.

(iv) $|A \cap B|=\infty$.

Proof. (i) It is convenient to put $\xi_{1}=\alpha^{-1}, \xi_{2}=\beta^{-1}$. Consider the sequence $\zeta=\{\alpha, \beta, 2 \alpha, 2 \beta, 3 \alpha, 3 \beta, \ldots\}$. It suffices to show that if $M \geq 1$ is any integer and there are $N_{M}$ members of $\zeta<M$, then $N_{M+1} \geq N_{M}+1$. The number of $n>0$ satisfying $n \alpha<M$ is $\left\lfloor M \xi_{1}\right\rfloor$, and the number of $n>0$ satisfying $n \beta<M$ is $\left\lfloor M \xi_{2}\right\rfloor$. So $N_{M}=\left\lfloor M \xi_{1}\right\rfloor+\left\lfloor M \xi_{2}\right\rfloor$. Now

$$
M \xi_{1}-1<\left\lfloor M \xi_{1}\right\rfloor<M \xi_{1}, \quad M \xi_{2}-1<\left\lfloor M \xi_{2}\right\rfloor<M \xi_{2} .
$$

Adding, $(3 M / 2)-2<N_{M}<3 M / 2$. If $M=2 t$ is even, then $3 t-2<N_{M}<3 t$, so $N_{M}=3 t-1$, and then $3 t-1 / 2<N_{M+1}<3 t+3 / 2$, so $N_{M+1} \in\{3 t, 3 t+1\}$. Thus $N_{M+1}-N_{M} \in\{1,2\}$. If $M=2 t+1, M+1=2 t+2$, we obviously also get $N_{M+1}-N_{M} \in\{1,2\}$, proving (i).

(ii) Since each of $A$ and $B$ is strictly increasing, $N$ can appear at most once in each.

(iii) Follows immediately from the fact that $\alpha<\beta$.

(iv) We have to show that $N_{M+1}-N_{M}=2$ is assumed for infinitely many $M \in \mathbb{Z}_{\geq 0}$. If $N_{M+1}-N_{M}=1$ for all large $M$ then a simple density argument shows that $\xi_{1}+\xi_{2}=1$, a contradiction.

Lemma 3. $\Delta\lfloor n \beta\rfloor=1$ implies

$$
\Delta\lfloor(n-2) \beta\rfloor=\Delta\lfloor(n-1) \beta\rfloor=\Delta\lfloor(n+1) \beta\rfloor=\Delta\lfloor(n+2) \beta\rfloor=2 .
$$

Proof. We have $\Delta\lfloor n \beta\rfloor=1$ if and only if $N<n \beta<N+1<(n+1) \beta<N+2$ for some $N \in \mathbb{Z}_{>0}$. Since the fractional parts $\{n \beta\}_{n>1}$ are dense in the reals (Kronecker's Theorem), this inequality holds for infinitely many pairs of integers $(n, N)$. Since $1.74<\beta<1.75$, we then have $N+3<(n+2) \beta<N+4<$ $N+5<(n+3) \beta<N+6$. Then $\Delta\lfloor(n+1) \beta\rfloor=\Delta\lfloor(n+2) \beta\rfloor=2$. We also have $\Delta\lfloor n \beta\rfloor=1$ if and only if $N-1>(n-1) \beta>N-2>N-3>(n-2) \beta>N-4$, so $\Delta\lfloor(n-2) \beta\rfloor=\Delta\lfloor(n-1) \beta\rfloor=2$.

Lemma 4. If $\Delta\lfloor n \alpha\rfloor=2$, then $\Delta\lfloor(n+i) \alpha\rfloor=1$ for at least all $i \in\{1, \ldots, 11\}$.

Proof. Follows from the fact that $\left\lfloor\{\alpha\}^{-1}\right\rfloor=12$, where $\{x\}$ denotes the fractional part of $x$.

Definition 1. For any real number $x$ and any $n \in \mathbb{Z}_{\geq 0}, \Delta\lfloor n x\rfloor$ is called an $x$-difference. 
Lemma 5. For $n, r \in \mathbb{Z}_{\geq 1}$, let

$$
\lfloor(n+r) \beta\rfloor-\lfloor n \beta\rfloor=\lfloor(n+r) \alpha\rfloor-\lfloor n \alpha\rfloor=t .
$$

Then $r \leq 2, t \leq 3$; and $r=2$ with $t=3$ is achieved.

Proof. We wish to maximize $r$. If any two consecutive $\beta$-differences are 2 , then the corresponding $\alpha$-differences cannot be 2 by Lemma 4 . So one of the two consecutive $\beta$-differences must be 1 . The corresponding $\alpha$-difference is then also 1 by Corollary 1 . The next $\beta$-difference is then necessarily 2 (Lemma 3 ), and the next $\alpha$-difference can be 2 . Then the next $\beta$-difference is still 2 , but the corresponding $\alpha$-difference is 1 . Thus $r \leq 2, t \leq 3$; and $r=2$ with $t=3$ in (5) is achieved, for example for $n=11$.

Lemma 6. Let $\left(a_{n}, b_{n}\right) \mathcal{T}$. Then $\left(a_{n}-t, b_{n}-t\right)=\left(a_{m}, b_{m}\right) \in \mathcal{T}$ for no $t>3$.

Proof. Follows immediately from Lemmas 3, 4 and 5.

\section{Proof of the Main Theorem}

We need to show $\mathcal{P}=\mathcal{T}$. Since FrEAK is acyclic, it suffices to show two things: Any move from any position in $\mathcal{T}$ results in a position in $\overline{\mathcal{T}}$; and from any position in $\overline{\mathcal{T}}$, there exists a move to a position in $\mathcal{T}$.

We precede these two aspects with a notation and a proposition.

Notation 1. For every $n \in \mathbb{Z} \geq 0$, let $d_{n}:=b_{n}-a_{n}$.

Lemma 7. (i) For every $n \in \mathbb{Z} \geq 0, d_{n+1}-d_{n} \in\{0,1\}$.

(ii) $d_{n}$ is a nondecreasing function of $n$.

(iii) $\cup_{n \geq 0} d_{n}=\mathbb{Z}_{\geq 0}$.

Proof. (i) We have, $d_{n+1}-d_{n}=\Delta\lfloor n \beta\rfloor-\Delta\lfloor n \alpha\rfloor$. By (3), $\Delta\lfloor n \alpha\rfloor \in\{1,2\}$. If $\Delta\lfloor n \alpha\rfloor=1$, then $\Delta\lfloor n \beta\rfloor \in\{1,2\}$. If $\Delta\lfloor n \alpha\rfloor=2$, then $\Delta\lfloor n \beta\rfloor=2$ by Lemma 1 .

(ii) It follows immediately from (i) that $d_{n}$ is nondecreasing.

(iii) The fact that the multiset $\cup_{n \geq 0} d_{n}$ contains every nonnegative integer also follows immediately from (i).

Any move from any position in $\mathcal{T}$ results in a position in $\overline{\mathcal{T}}$. Let $\left(a_{n}, b_{n}\right) \in \mathcal{T}$, $n \geq 1$. We have to show that $\left(a_{n}, b_{n}\right) \rightarrow\left(a_{m}, b_{m}\right) \in \mathcal{T}$ for no $m \geq 0$. For $t \in\{1,2,3\},\left(a_{n}, b_{n}\right) \rightarrow\left(a_{n}-t, b_{n}-t\right)$ is blocked by (I). For $t>3,\left(a_{n}-t, b_{n}-\right.$ $t) \rightarrow\left(a_{m}, b_{m}\right)$ is impossible (Lemma 6$)$. Since $A$ and $B$ are strictly increasing, a move of type B cannot lead from $\mathcal{T}$ to $\mathcal{T}$.

From any position in $\overline{\mathcal{T}}$, there exists a move to a position in $\mathcal{T}$. Suppose $(x, y) \in$ $\overline{\mathcal{T}}, 0 \leq x \leq y$. We first deal with the case $x=y:=t$. For $t=1,(t, t)=(1,1)$ is in $\mathcal{T} ;(2,2) \rightarrow(0,0)$ is not blocked since $2 \notin B$. Also $(3,3) \rightarrow(2,3) \in \mathcal{T}$ is not blocked: it is a move of the form (II)(a). For $t>3$, taking $(t, t)$ is never 
blocked. Moreover, $(0, y) \rightarrow(0,0)$ and $(1, y) \rightarrow(1,1)$ are not blocked. We may thus assume $1<x<y$. Then $x=a_{n}=b_{m}$ implies $n>m$, since $\beta>\alpha$, so $B$ increases at least as fast as $A$ (CF Lemma 2(iii)).

Since $A, B$ cover the nonnegative integers (Lemma 2(i)), we have either (i) $x=a_{n}$ or (ii) $x=b_{n}$ for some $n \in \mathbb{Z}_{\geq 0}$. Of course Lemma 2(iv) implies that $x=a_{n}=b_{m}$ for infinitely many $n>m>1$.

(i) $x \in B$, say $x=b_{m}$.

(i1) $x \notin A$. Then the Nim move $y \rightarrow a_{m}$ is a non blocked move of the form (II) (c).

(i2) $x \in A$, say $x=a_{n}$. We have $1<m<n$.

(i21) $y>b_{n}$. Then do $y \rightarrow b_{n}$. This move is of the form (II)(b). It is not blocked, since $b_{n}>x=a_{n}$.

(i22) $y<b_{n}$. We consider two cases.

1. $y \in B$, say $y=b_{k}$. Then $k<n$, so can make the (II)(a) move $x \rightarrow a_{k}$.

2. $y \notin B$. Then move $y \rightarrow x_{m}$. It is an unblocked move of the form (II)(c).

(ii) $x \in A$, say $x=a_{n}$. The case where also $x \in B$, say $x=b_{m}$, was dealt with in (i2) above, so we may assume $x \notin B$.

(ii1) $y>b_{n}$. Then move $y \rightarrow b_{n}$. This Nim move is not blocked, since $b_{n}>$ $a_{n}=x$. The move is of the form (II)(b).

(ii2) $y<b_{n}$. If $y \in B$, say $y=b_{k}$, then we have $k<n$, so we can move $x \rightarrow a_{k}$, as in (i22)1. So we may assume $y \notin B$. We have $1<a_{n}=x<y<b_{n}$. Let $d:=y-x=y-a_{n}<b_{n}-a_{n}=d_{n}$. By Lemma 7(iii), there exists $k<n$ such that $d_{k}=d$, that is, $b_{k}-a_{k}=y-a_{n}$, so $y-b_{k}=a_{n}-a_{k}:=t$. Then the Wythoff move $(x, y) \rightarrow\left(a_{n}-t, y-t\right)=\left(a_{k}, b_{k}\right) \in \mathcal{T}$ is not blocked, even if $t \in\{1,2,3\}$, since $y \notin B$.

\section{A Polynomial Winning Strategy}

Given any game position $(x, y)$ of FrEAK subject to (2), it obviously suffices to know whether $x \in A, x \in B, y \in A, y \in B$. The proof of Theorem 1 then enables us to win if $(x, y) \in \overline{\mathcal{T}}$.

Theorem 2. The computations to determine whether or not any of $x \in A$, $x \in B, y \in A, y \in B$ holds is polynomial-time in the succinct input size $\log x+$ $\log y=\log x y$ of any input game position $(x, y), 1 \leq x \leq y$.

Proof. Since $\alpha$ is irrational and $1<\alpha<2$,

$x=\lfloor n \alpha\rfloor \Longleftrightarrow x<n \alpha<x+1 \Longleftrightarrow \frac{x}{\alpha}<n<\frac{x+1}{\alpha} \Longleftrightarrow\left\lfloor\frac{x+1}{\alpha}\right\rfloor=\left\lfloor\frac{x}{\alpha}\right\rfloor+1$.

Therefore either $x=\lfloor n \alpha\rfloor=a_{n}$, where $n=\lfloor(x+1) / \alpha\rfloor$, or else, by Lemma 2(i), $x=\lfloor n \beta\rfloor=b_{n}$, where $n=\lfloor(x+1) / \beta\rfloor$. 
Since also $1<\beta<2$, we can compute the same way whether $y=\lfloor n \beta\rfloor$, together with the multiplier $n$ and/or whether $y=\lfloor n \alpha\rfloor$ with its multiplier $n$. These computations require that $\alpha$ and $\beta$ be computed to a precision of only $O(\log y)$ digits. Once we made these poly-time computations, we make the appropriate move prescribed in sub-steps of (i) or (ii) of the proof of Theorem 1.

\section{An Alternate Polynomial Winning Strategy}

We now present a strategy that depends on two exotic numeration systems. Recall that any positive irrational $\alpha$ can be expanded in a simple continued fraction:

$$
\alpha=a_{0}+\frac{1}{a_{1}+\frac{1}{a_{2}+\frac{1}{a_{3} \ldots}}}:=\left[a_{0}, a_{1}, a_{2}, a_{3} \ldots\right],
$$

where $a_{0} \in \mathbb{Z}_{\geq 0}, a_{i} \in \mathbb{Z}_{\geq 1}, i \geq 1$. The convergents of the continued fraction are the rationals $p_{n} / q_{n}=\left[a_{0}, \ldots, a_{n}\right]$, and they satisfy the recurrences (see e.g., [12], ch. 10):

$$
\begin{array}{ll}
p_{-1}=1, p_{0}=a_{0}, p_{n}=a_{n} p_{n-1}+p_{n-2} & (n \geq 1), \\
q_{-1}=0, q_{0}=1, q_{n}=a_{n} q_{n-1}+q_{n-2} & (n \geq 1) .
\end{array}
$$

For the case $a_{0}=1$ (then $1<\alpha<2$ ), one of the numeration systems, the $p$-system, is spawned by the numerators of the convergents (see [9], [5]): Every positive integer $N$ can be written uniquely in the form

$$
N=\sum_{i \geq 0} s_{i} p_{i}, 0 \leq s_{i} \leq a_{i+1}, s_{i+1}=a_{i+2} \Longrightarrow s_{i}=0 \quad(i \geq 0) .
$$

Denote by $S, T$, the numeration systems based on the numerators of the convergents of the simple continued fraction expansion of $\alpha, \beta$, respectively. For any positive integer $N$, let $R_{S}(N), R_{T}(N)$ denote the representations of $N$ in the $S, T$ numeration systems, respectively. We say that $N$ is $S$-vile, $T$ vile if $R_{S}(N), R_{T}(N)$ respectively ends in an even number (possibly 0) of 0s. Analogously, $N$ is $S$-dopey, $T$-dopey if $R_{S}(N), R_{T}(N)$ respectively ends in an odd number of 0 s.

Note 1. The names "evil" and "dopey" are inspired by the evil and odious numbers, those that have an even and an odd number of 1's in their binary representation respectively. To indicate that we count 0 s rather than $1 \mathrm{~s}$, and only at the tail end, the "ev" and "od" are reversed to "ve" and "do" in "vile" and "dopey". "Evil" and "odious" were coined by Elwyn Berlekamp, John Conway and Richard Guy [1].

We notice that

$$
\alpha=[1,12,1,2,2,2, \alpha], \quad \beta=[1,1,2, \alpha] .
$$


The periodicities are of course a manifestation of Lagrange's Theorem ([12], ch. 10). For $\alpha$ we have $p_{0}=1, p_{1}=13, p_{2}=14, p_{3}=41, p_{4}=96, \ldots$. For $\beta$, $p_{0}=1, p_{1}=2, p_{2}=5, p_{3}=7, p_{4}=89, \ldots$. Also $s_{0} \leq a_{1}=1$, so $s_{0} \in\{0,1\}$ for both numeration systems. In Table 2 we exhibit $R_{S}(N)$ on the left-hand side and $R_{T}(N)$ on the right-hand side for the first few positive integers $N$.

Table 2. Representation of $1 \leq n \leq 15$ in the $S$ - (left) and $T$-system (right).

\begin{tabular}{|r|r|r||r||l|l|l|l|}
\hline 14 & 13 & 1 & $n$ & 7 & 5 & 2 & 1 \\
\hline 0 & 0 & 1 & 1 & 0 & 0 & 0 & 1 \\
0 & 0 & 2 & 2 & 0 & 0 & 1 & 0 \\
0 & 0 & 3 & 3 & 0 & 0 & 1 & 1 \\
0 & 0 & 4 & 4 & 0 & 0 & 2 & 0 \\
0 & 0 & 5 & 5 & 0 & 1 & 0 & 0 \\
0 & 0 & 6 & 6 & 0 & 1 & 0 & 1 \\
0 & 0 & 7 & 7 & 1 & 0 & 0 & 0 \\
0 & 0 & 8 & 8 & 1 & 0 & 0 & 1 \\
0 & 0 & 9 & 9 & 1 & 0 & 1 & 0 \\
0 & 0 & 10 & 10 & 1 & 0 & 1 & 1 \\
0 & 0 & 11 & 11 & 1 & 0 & 2 & 0 \\
0 & 0 & 12 & 12 & 1 & 1 & 0 & 0 \\
0 & 1 & 0 & 13 & 1 & 1 & 0 & 1 \\
1 & 0 & 0 & 14 & 2 & 0 & 0 & 0 \\
1 & 0 & 1 & 15 & 2 & 0 & 0 & 1 \\
1 & 0 & 2 & 16 & 2 & 0 & 1 & 0 \\
1 & 0 & 3 & 17 & 2 & 0 & 1 & 1 \\
1 & 0 & 4 & 18 & 2 & 0 & 2 & 0 \\
1 & 0 & 5 & 19 & 2 & 1 & 0 & 0 \\
1 & 0 & 6 & 20 & 2 & 1 & 0 & 1 \\
\hline
\end{tabular}

Comparing Tables 1 and 2, notice that, at least for the range $n \in[1,20]$ : $n \in A$ if and only if $n$ is $S$-vile; $n \in B$ if and only if $n$ is $T$-vile. This property holds in general - see [5], sect. 5. It follows immediately that the game rules of FrEAK, in terms of the $S$ - and $T$-numeration systems, can be stated as follows:

(I) (Restricted Wythoff move.) $(x, y) \rightarrow(x-t, y-t)$ for every $t \in\{1, \ldots, x\}$, except that this move is blocked if the following three conditions hold: (a) $t \in\{1,2,3\}, \quad$ (b) $x$ is $S$-vile, (c) $y$ is $T$-vile.

(II) (Restricted Nim move.) (a) $(x, y) \rightarrow(x-t, y)$ for any $0<t \leq x$; or

(b) $(x, y) \rightarrow(x, y-t)$ for any $0<t \leq y$; or

(c) $(x, y) \rightarrow(y-t, x)$ for any $0<t \leq y$ except that this move is blocked if $x$ is both $S$-vile and $T$-vile and $y$ is $T$-vile.

The computation whether $x$ or $y$ is $S$-vile or $T$-vile can obviously be done in poly-time in the input size $\log x y$ of any game position $(x, y)$. It follows that also the winning strategy based on the two numeration systems is poly-time. It 
has the advantage of avoiding the floor function and division, both of which are needed for our first winning strategy.

\section{$7 \quad$ Epilogue}

Preliminary Thoughts. We presented two polynomial-time winning strategies for a game on $m=2$ piles of tokens for which the $P$-positions constitute a single pair of integers $\left(A^{1}, A^{2}\right)$ (in contrast to [2] and [8]), $\left(A^{1}, A^{2}\right)$ satisfy $\left|A^{1} \cap A^{2}\right|=\infty$, but $\left|A^{1} \cup A^{2}\right|=\mathbb{Z}_{\geq 1}$. It appears to be a first such case.

FREAK, the name of the game, derives from FRactional BEAtty game. The terminology "vile" and "dopey" is inspired by the evil and odious numbers, those that have an even and an odd number of 1's in their binary representation respectively. To indicate that we count 0 s rather than $1 \mathrm{~s}$, and only at the tail end, the "ev" and "od" are reversed to "ve" and "do" in "vile" and "dopey". "Evil" and "odious" were coined by Elwyn Berlekamp, John Conway and Richard Guy while composing their famous book Winning Ways [1]. Urban Larsson suggested the particular values of $\alpha, \beta$ used in this work. A "fractional Beatty theorem" was recently proved by Peter Hegarty [13] (following a suggestion of mine). In previous papers we have shown that a judicious choice of numeration systems can improve the efficiency of winning strategies of various games, much as data structures in Computer Science. In the present paper, numeration systems are the tool used uniformly for both formulating and analyzing FREAK.

\section{Further questions.}

(1) Extend the above results to an infinite set of fractional Beatty games, for example, for $\alpha=\ell \varphi /(2 k+1), \beta=\ell \varphi^{2} /(2 k+1), k, \ell$ any fixed positive integers.

(2) Are there "simpler" game rules for the same set of $P$-positions considered here?

(3) A move $R=\left(r_{1}, \ldots, r_{m}\right) \neq(0, \ldots, 0)$ in an $m$-pile subtraction game is invariant if $R$ can be made from every game position $\left(s_{1}, \ldots, s_{m}\right)$ for which $s_{i}-r_{i} \geq 0$ for $i=1, \ldots, m$. An $m$-pile subtraction game is invariant if all its moves are invariant. Otherwise the game is variant. The move rules for FREAK are obviously variant. Duchêne and Rigo [4] conjectured that for $m=2$, given any two complementary Beatty sequences $A, B$, there exists an invariant game with $(A, B) \cup\{(0,0)\}$ as its $P$-positions. This conjecture was proved in [15]. Is there an invariant game with the $P$-positions presented in sect. 2 above?

(4) More generally, can the invariance theorem proved in [15] be extended in the following sense: Is there a nontrivial subset of non-complementary Beatty sequences $A, B$, for which there always exists an invariant game with $(A, B) \cup$ $\{(0,0)\}$ as its $P$-positions?

(5) Let $r, t \in \mathbb{R}_{>0}$. The equation $\alpha^{-1}+(\alpha+t)^{-1}=r$ has the positive solution $\alpha=\left(2 r^{-1}-t+\sqrt{t^{2}+4 r^{-2}}\right) / 2$. For every set of values $(r, t) \in \mathbb{R}_{>0}^{2}$ for which $\alpha$ is irrational one can define, in principle, an $(r, t)$-Beatty game. So there is a continuum of such games. If $r$ and $t$ are restricted to be rational we get a denumerable number of games. (One can even consider such games when 
$\alpha$ is rational, see [7].) For example, for $r=3 / 2, t=2, \alpha=(\sqrt{13}-1) / 3$ (so $2 / 3<\alpha<1)$, and $\beta=\alpha+2=(\sqrt{13}+5) / 3$. It may be of interest to formulate game rules for a game whose $P$-positions are $\cup_{n \geq 0}\left(a_{n}, b_{n}\right)$, where $a_{n}=\lfloor n \alpha\rfloor$, $b_{n}=\lfloor n \beta\rfloor$. In this game there are infinitely many integers that are repeated (at most twice) in $\left\{a_{n}\right\}_{n \geq 0}$, in addition to $|A \cap B|=\infty$. But there is the nice property that $b_{n}=a_{n}+2 n$ for all $n \geq 0$, as can be seen in Table 3 below.

Table 3. The first few terms of the $P$-positions $\left(a_{n}, b_{n}\right)$.

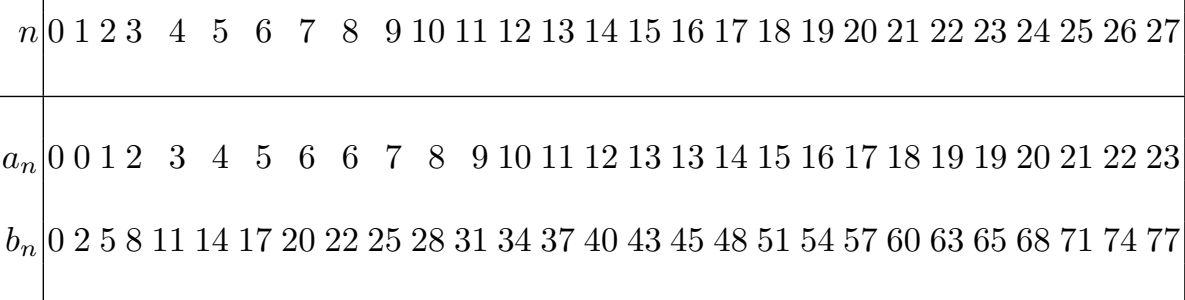

(6) Investigate the Sprague-Grundy function of fractional Beatty games in an attempt to give a poly-time winning strategy for playing them in a sum.

(7) Consider take-away games on $m>2$ piles, where the $m$ sequences $A^{1}, \ldots, A^{m}$ constituting the $P$-positions do not split $\mathbb{Z}_{>1}$. $\mathbb{Z}_{\geq 1}$

(8) Consider partizan take-away games where the $P$-positions do not split

(9) Investigate Fractional Beatty games for misère play.

Acknowledgment. Thanks to Urban Larsson, for his useful comments at the beginning of this work.

\section{References}

[1] E. R. Berlekamp, J. H. Conway and R. K. Guy, Winning Ways for your Mathematical Plays, Vol. 1-4, A K Peters, Wellesley, MA, 2nd edition: vol. 1 (2001), vols. 2, 3 (2003), vol. 4 (2004).

[2] I. G. Connell, A generalization of Wythoff's game, Canad. Math. Bull. 2 (1959) 181-190.

[3] E. Duchêne and M. Rigo, Amorphic approach to combinatorial games: the Tribonacci case, Theor. Inform. Appl. 42 (2008) 375393.

[4] E. Duchêne and M. Rigo, Invariant games, Theoret. Comput. Sci. 411 (2010) 3169-3180.

[5] A. S. Fraenkel, How to beat your Wythoff games' opponent on three fronts, Amer. Math. Monthly 89 (1982) 353-361. 
[6] A. S. Fraenkel, The Raleigh game, Integers, Electr. J. of Combinat. Number Theory $\mathbf{7 ( 2 0 )}$, special volume in honor of Ron Graham, \#A13, 11 pp., 2007, http://www.integers-ejcnt.org/vol7(2).html

[7] A. S. Fraenkel, The rat game and the mouse game, to appear in Games of No Chance 4 .

[8] A. S. Fraenkel and I. Borosh, A generalization of Wythoff's game, J. Combinatorial Theory (Ser. A) 15 (1973) 175-191.

[9] A. S. Fraenkel, J. Levitt and M. Shimshoni, Characterization of the set of values $f(n)=[n \alpha], n=1,2, \ldots$, Discrete Math. 2 (1972) 335-345.

[10] R. Graham and K. O'Bryant, A discrete Fourier kernel and Fraenkel's tiling conjecture, Acta Arith. 118 (2005) 283-304.

[11] V. Gurvich, Further generalizations of Wythoff's game and minimum excludant function, Rutgers Research Report RRR 16-2010, 2010 .

[12] G. H. Hardy and E. M. Wright, An Introduction to the Theory of Numbers, 6th ed., Oxford, 2007.

[13] P. Hegarty, On $m$-covering families of Beatty sequences with irrational moduli, 2011, preprint.

[14] U. Larsson, Restrictions of $m$-Wythoff Nim and $p$-complementary Beatty sequences, to appear in Games of No Chance-4.

[15] U. Larsson, P. Hegarty and A. S. Fraenkel, Invariant and dual subtraction games resolving the Duchêne-Rigo conjecture, Theoretical Computer Science 412 (2011) 729-735.

[16] K. O'Bryant, A generating function technique for Beatty sequences and other step sequences, J. Number Theory 94 (2002) 299-319.

[17] Y. Tanny and A. S. Fraenkel, Wythoff-like games, in preparation. 